\title{
Implementation of Cmos Camera Device Driver and Wifi Technology on S3c2440 Using Linux
}

\author{
M.Sumalatha ${ }^{1}$, D.Jaya Kumar ${ }^{2}$ \\ ${ }^{1}$ Student, ${ }^{2}$ Assistant Professor \\ ${ }^{l}$ ECE Department, Mallareddy Institute Of Engineering And Technology. \\ ${ }^{2}$ ECE Department, Mallareddy Institute Of Engineering And Technology. \\ Hyderabad, Ap, India. ${ }^{1}$, Hyderabad, Ap., India ${ }^{2}$.
}

\begin{abstract}
With the processing of CMOS technology, the technology of video acquisition based on CMOS is becoming a new trend. However, many CMOS camera chip is not supported by the newest Linux kernel yet. The environmental image acquirement and the Wi-Fi transmission system are studied and designed. In this paper, the method of designing the CMOS camera driver based on S3C2440 developing board with the embedded Linux environment is introduced and adds some components such as a USB Wi-Fi adapter. SCCB is a distinguishing feature of OV series CMOS chips. S3C2440 provides a camera interface, and the camera driver is designed based on it. The library and the utilities are compiled, and of images got from CMOS camera to the Wi-Fi mobile phone has been realized by means of programming.
\end{abstract}

\section{Introduction}

Comparing with traditional CCD image sensor, CMOS image sensors[1] using CMOS technology can integrate the pixel array and peripheral circuits (such as the image sensor core, single-clock, all the sequential logic, programmable functions and A / D converter) in a chip, with a small size, light weight, low power consumption, programming convenience, easy to control and so on.

Embedded Linux [4] is a better embedded operation system, which has portable, strong network function and excellent GNU compile tools as well as free open source characters. S3C2410 is a 32 bits embedded processor that based on an ARM920T core[3] with integrated MMU and abundant internal resources. Because of low cost, high performance and the Video4linux (short for V4L) support on Linux program, CMOS camera [2] is easy to integrate to embedded system. Wi-Fi technique is a short distance wireless communication with dominant position which is exclusive certificated, and it has been widely used in mobile phones, personal computer and peripherals at present. This paper introduce image acquirement function based on embedded Linux system, $[5,6]$ and transmit the picture got from CMOS camera to the Wi-Fi mobile phone on the environment of Wi-Fi that has been constructed.

\section{A. Software design module}

\section{System Design Model}

An operating system (OS) is software, consisting of programs and data that runs on computers and manages the computer hardware and provides common services for efficient execution of various application software. We cannot get S3C2440 microcontroller individually. We will get it in the form of FRIENDLY ARM board else, we can call it as MINI 2440 board. In order to work with ARM 9 micro controllers we require 3 things. They are as follows.

Boot loader: The main functionality of boot loader is to initialize all the devices that are present on the motherboard of MINI 2440 and at the same time to find out whether any problem or any other fault is there in the devices that are present on that motherboard of MINI 2440.The other feature of the boot loader is to find out what are the different operating systems that are present in the standard storage devices and to show it on to the display device so that user can select between the operating systems into which he wants to enter. One other feature of the boot loader is lo load operating system related files byte by byte into the temporary memory like RAM. In our current project, we are using boot loader like Super vivi, which is MINI 2440 specific.

Kernel: The core part of an operating system we can call like kernel. Operating system will perform its functionalities like File management, Process management, Memory management, Network management and Interrupt management with the help of the kernel only. Kernel holds the device related drivers that are present on the motherboard. FRIENDLY ARM board supports for operating systems like SYMBIAN, ANDROID, EMBEDDED LINUX, WINCE. However, in all these operating systems EMBEDDED LINUX will provide high security to drivers and files. Therefore, in our current project we are making use of kernel of EMBEDDED 
LINUX with which device related drivers that are present on the motherboard of FRIENDLY ARM board will automatically come when we load EMBEDDED LINUX related kernel.

Root File System: File system will tell how files arrangement there inside the internal standard storage devices. In embedded Linux, kernel treats everything as a file even the input and output devices. In embedded Linux, Root is the parent directory it contains other sub directories like dev, lib, home, bin ,sbin ,media ,mnt ,temp ,proc, etc, opt and etc. According to our application, we will interface some external devices also. All the devices means internal devices that are present on the motherboard of MINI 2440 will get their corresponding drivers when we load Embedded Linux related kernel. However, these device drivers require micro controller related header files and some other header files, which will be present in the lib directory, which is present in the root directory. In addition, the devices related drivers would be present in the dev directory, which is again present in the root directory. Therefore, whenever we will load the Root File System then we will get different directories, which will be helpful to the kernel. So compulsorily, we need to load the Root File System. MINI 2440 specific Root File System is Root Qtopia.

The essential programs that are required in order to work with MINI 2440 like Boot loader, Embedded Linux related Kernel, Root File System will be loaded into the NOR flash which is present on the MINI 2440 board itself. The program related with the application will be loaded into NAND flash, which is also present on the MINI 2440 board itself. By using bootstrap switch that is present on the MINI 2440 will help the user to select either NOR or NAND flash. After that by using DNW tool we can load Boot loader, Embedded Linux related kernel and Root File System into NOR flash by using USB cable and the application related program into NAND flash.Once loading everything into MINI 2440 board it starts working based on the application program that we have loaded into the NAND flash.

The ARM 32 bit Microcontroller has feature of image/video processing by using various features and classification algorithms have been proposed for pedestrian detection. It overcomes the performance in terms of sensors and hardware cost is also too high. So, our design Embedded system that detects person just as they enter the camera view, with low false alarm rate and high speed. This system captures the eye from web camera connected to ARM microcontroller through USB and the image is processed by using image processing technique. Image processing is any form of signal processing for which the input is an image, such as a photograph or video frame, the output of image processing may be either an image or a set of characteristics or parameters related to the image.

\section{B. Hardware design module BLOCK DIAGRAM}

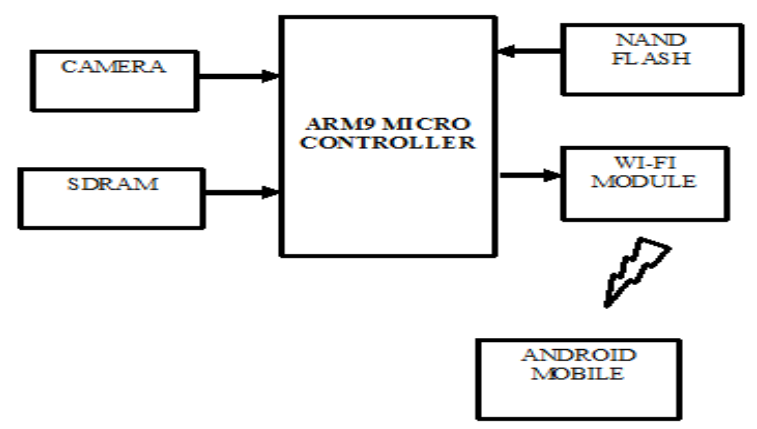

Figure: hardware implementation block diagram.

With the processing of CMOS technology, the technology of video acquisition based on CMOS is becoming a new trend. However, many CMOS camera chip is not supported by the newest Linux kernel yet.

The system uses SAMSUNG S3C2440 as a microprocessor, the camera equipment is OV9650. SAMSUNG S3C2440 uses 16/32 bit ARM920T RISC technology for the core. Its main Frequency is $400 \mathrm{M} \mathrm{Hz}$. It provides a camera interface (camif) to support camera. There are two models for camif to transmit data with DMA controller: one is called Preview mode, which transform the image data sampling from the camera interface into the RGB format, and transfer it to the SDRAM under control of the DMA; the other is called code mode, which transmits the image data to the SDRAM in YCbCr4:2:0 or 4:2:2 format. Software platform is embedded Linux OS. In this application we are going to use the preview mode.

ARM9 is continuously monitoring the video by using CMOS camera. For the ARM9 board USB based Wi-Fi module is connected and sending video continuously through Wi-Fi. An android mobile will be used to create a Wi-Fi hotspot which will create the Wi-Fi network. After the Wi-Fi network is created, ARM9 board will connect to that network using its own Wi-Fi module, and all the other clients (computers or laptops or mobiles) 
with Wi-Fi capability can connect to the same network and will access the ARM9's IP address on the browser to see the video.

\section{Experimental Results}

With the continuous progress of CMOS technology, CMOS image sensors are more and more high resolution, low-cost, small size and easy programming, its application will become increasingly widespread. In this paper, the method of designing the CMOS camera driver based on S3C2440 developing board and Image acquirement in embedded linux is realized.
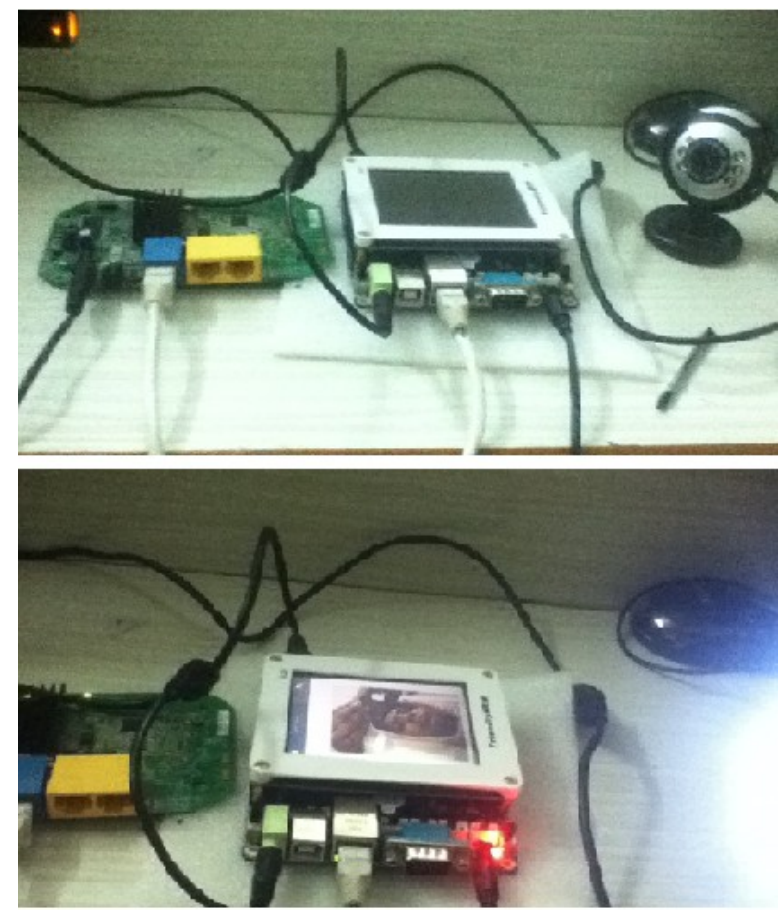

Figure: experimental set-up implementation.

We constructed the working environment of Wi-Fi, and completed the file transmission between embedded Linux platform and Wi-Fi mobile phone by Wi-Fi OBEX protocol and Object PUSH service. We realize remote surveillance by embedded system and wireless transmission.

\section{Conclusion}

The project "implementation of CMOS camera device driver and Wi-Fi technology on s3c2440 using Linux" has been successfully designed and tested. It has been developed by integrating features of all the hardware components and software used. Presence of every module has been reasoned out and placed carefully thus contributing to the best working of the unit. Secondly, using highly advanced ARM9 board and with the help of growing technology the project has been successfully implemented.

\section{References}

[1] El GamalA.Eltoukhy H.CMOS image sensors[J].USA Circuits and Devices Magazine IEEE, 2005, 21(3): 6-20

[2] Yuan Weiqi, Tang Yonghua. Application of SCCB of OmniVision camera in DSP[J]. Chinese Journal of Scientific Instrument.2006_27_6_1687-1688. (in Chinese)

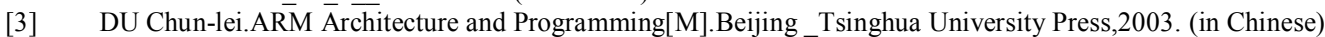

[4] Zhang Da-bo. Principles, Design and Application of Embedded System[M].Beijing_Machinery Industry Press_2004. (in Chinese)

[5] Samsung. S3C2440A 32Bit CMOS Microcontroller User's Manual[Z]. Samsung Electronics Corp, 2003.

[6] Jonathan C, Alessandro R, Greg K. Linux device drivers[M].3rd ed. Sebastopl, CA:O’ Reilly \& Associates, 2006:324-327.

[7] Daniel P, Cesati M. Understanding the Linux kernel [M]. 3rd ed. 